\title{
The case for multidisciplinary research in primary care
}

A.S. Raghunath and A. Innes, Department of Primary Care Medicine, University of Hull, Hull, UK

In this paper we attempt to explore the meaning, understanding, usefulness and reality of multidisciplinary research in primary care and provide examples. We debate the issues around roles of people in multidisciplinary research, the value of such research and its application to patient care. We also discuss the perceived threats and opportunities to multidisciplinary research, possible reasons for such perceptions, funding, and research governance, education and training issues. We then go on to consider the emerging relationship between primary care organizations (PCOs) and primary care research teams. We specifically address the activities of research general practices and their implications in terms of contributing to multidisciplinary research and training. We question the traditional model of academic research; raise awareness and need for grassroots primary care research and the importance of capacity building through a multidisciplinary model. We have tried to suggest examples and models of multidisciplinary research that can be seamless, include a range of practices and health professionals, and performs research that is contextual, pragmatic and directly beneficial to patients. We feel that for a successful multidisciplinary research workforce in primary care to become a reality, several key areas need to be addressed. Perhaps the most important of these may involve a conceptual change in thinking by all concerned in that we believe that research in primary care should become an integral part of routine primary care in the same way as health professionals routinely see patients or conduct audits. This could, however, only happen if there is commitment, support and a vision for the future of primary care research from decision makers. Local research networks and PCOs need to work closely together in identifying, nurturing and maintaining multidisciplinary research interest.

Key words: multidisciplinary research; primary care; primary care organizations; research networks; research practice; research priorities

\section{Introduction}

Multidisciplinarity has become a popular concept (Thomas and While, 2001) in current primary care research and development circles and yet the reality of such a concept is still in its infancy; its meaning not yet fully defined. In this article, we argue and consider multidisciplinary issues, how we encourage and support multidisciplinary research

Address for correspondence: Dr A.S. Raghunath, St Andrews Group Practice, Marmaduke St Health Centre, Hull and Honorary Research Fellow, Department of Primary Care Medicine, University of Hull, UK. Email: Raghu@nath.freeserve.co.uk and how we achieve and assess the success of such multidisciplinary research (Figure 1).

\section{Multidisciplinary research - background}

In delivering clinical service commitments, primary care has increasingly developed a multidisciplinary approach. For example, the care of a patient with diabetes in general practice would be co-ordinated through the combination of a receptionist, practice nurse, chiropodist, dietician, and general practitioner (GP). Expert opinion from a hospital consultant would be obtained as necessary. Such a team approach is equally applicable to other clinical conditions, for example asthma, 
- Multidisciplinary research - a rationale.

- Multidisciplinary research -

Implementation/practical issues.

- Illustrations from our experience.

- Assessment and governance.

- Conclusions and recommendations.

Figure 1 Summary

hypertension and heart disease. We believe that multidisciplinary research in primary care should build on this already established model of clinical teamwork and should seek to include the full range of health and social care workers delivering primary care services. Involvement of patients in a multidisciplinary research team as opposed to just being subjects in research is a concept that is starting to be recognized as being very important to the success of the research process (Coulter, 1999; Goodare and Lockwood, 1999; Williamson, 1999). It might also help in identifying relevant research topics in primary care.

The World Health Organization suggests that there is a need for a body of nurses who are able to undertake research: "A sizeable number of nurses and midwives will need to be enabled to develop research skills, in order to participate as equal members in multidisciplinary research teams' (WHO, 1994). Similarly in general practice GPs have been encouraged by their leaders to become more involved in primary care research (Carter and Thomas, 1997). Although it is recognized that the majority of clinical contacts take place in primary care, the majority of clinical research takes place in secondary care settings (Hobbs, 1997).

Since the publication of the Mant report (Mant, 1997) there has been a visible increase in primary care research activity. Research networks, research practices and more available funding to support and develop individuals have all contributed to that development, as has an increase in the number of academic posts relevant to primary care (Beasley, 1993; Radda, 1998; Shaw and Carter, 2000).

Several years into this expansion in research and development, there remain, however, a number of unresolved questions: Is the investment in primary care research based on the Mant report value for money? Is the type of research that is being undertaken in primary care relevant? (Kernick et al., 1999). Is new capacity really being built or is it that old capacity is simply being expanded? Is there excessive pressure on primary care health professionals to undertake the dual role of research and service commitment?

\section{Multidisciplinary research - a rationale}

We acknowledge the complexity of primary care by working as multidisciplinary health care teams but so far we have largely worked independently in our research efforts. Bringing together the same multidisciplinary team to consider research will challenge professional values and assumptions and identify the research questions relevant to a multidisciplinary environment. Such an approach truly develops a research-minded workforce capable of critically appraising research more generally. Where such research bears fruit it will be more appealing and relevant to its audience and therefore more likely to make the important leap from research into practice (Whitford et al., 2000). Our definition of multidisciplinary research therefore would be one that brings together all those practitioners involved in the service provision of primary health care. All too often in the past, primary care has been used as a data collection resource for external researchers. Multipractice and multidisciplinary collaboration from the outset has the potential of ensuring the quality and success of a research project. Problems such as recruiting an adequate number of participants for a randomized trial (van der Windt et al., 2000) would be minimized through commitment, enthusiasm and ownership of the project by the entire team (Figure 2).

It is now becoming clearer that primary care multidisciplinary research in order to be successful has to be more context based and pragmatic. For instance, randomized controlled trials in primary care have to be both rigorous and pragmatic (Hippisley-Cox and Pringle, 1997). In other words, whilst academic rigour is important, the research needs to reflect the complex and messy nature of primary care. Traditional research paradigms may be less relevant and we may need to look, for Press 
- Majority of consultations take place in primary care

- Most decisions and actions are not evidence based

- Most consultations lead to a multidisciplinary care approach

- Research likely to be pragmatic and context based

- Research likely to be successful due to shared ownership and patient involvement

- Research findings likely to be quickly disseminated and implemented

- Likely to be less expensive

Figure 2 Rationale for multidisciplinary research in primary care

example, to the emerging science of chaos and complexity to find more meaningful ways of exploring our world in primary care (Griffiths and Byrne, 1998). There is of course a role for traditional quantitative and qualitative methods. However quantitative methods need to be much more pragmatic and reflect the real world of primary care rather than conforming to strict linear paradigms of 'modern' research and the statistical methods employed have to be sensitive to this approach. Qualitative research methods are often more appropriate to the frequently nebulous areas that primary care research must address. Research has to take into account the complex environment of primary care and hence is best developed within primary care by primary care professionals supported as needed by expert research resources.

\section{Threats and opportunities in multidisciplinary research}

Despite some evidence of recent growth in the numbers of individual practitioners undertaking research in primary care, we are not aware that there has been any substantial increase in capacity building of primary care multidisciplinary research teams. This may be of particular importance to the involvement of nursing and other team members in primary care research since their professional links may be less well developed (McKenzie, 1991; Mooney, 1996). The reasons for this may be several and complex; some practical and others less so (Figure 3). GPs for instance come from a background of professional training, experience, employment status and authority that may provide the confidence, resources, skills and opportunities to take part in research generally and multidisciplinary research in particular. However, they may be held back by lack of time and other work and family commitments. Nurses, particularly working in general practice may not have the 'power' to develop research in their practices. Like GPs, nurses share an educational heritage where research has not traditionally been integral to their professional development. Both do, however, share a focus on teamworking and developing multidisciplinary research teams might be seen as an ideal solution to develop research capacity in primary care.

Primary care in general, but nurses in particular have often been relegated to the task of data collectors for research conducted by secondary care. Perhaps this might have created an unhelpful role model for nurses and GPs in respect of research.

Primary care research has at times appeared remote from the reality of clinical practice. Grass root GPs and other primary care health professionals may and do have their own research questions that can be very different to those of the NHS primary care research agenda (Kernick et al., 1999).

Reviews of the development of nursing research 


\begin{tabular}{|ll}
\hline Threats & \multicolumn{1}{c}{ Opportunities } \\
Employment status & Existing PHC teams \\
Employer support & Diverse skills \\
Service commitment & NHS plan \\
Time & Resource opportunities \\
Confidence & Research practices \\
Educational background & Research networks \\
Training & Academic departments \\
Role confusion & PCOs \\
& Patients
\end{tabular}

Figure 3 Threats and opportunities

also highlight problems in particular with relation to the use of research findings in practice: the theorypractice gap. For example, English (English, 1994) identifies, in a review of research on the subject, seven reasons why research is not utilized in practice: nurses do not read relevant literature and are not convinced of the practical use of research findings; there is a lack of high quality research appropriate for utilization in practice; there is too much research, much of which produces conflicting findings; there is a time delay between the generation of research findings and application which is exacerbated by problems in communication between researchers and practitioners, and there is a lack of a research culture in colleges of nursing and amongst nurse educationalists. In addition Hicks (Hicks, 1995) found that nurse researchers were reluctant to submit their research findings for publication, providing another impediment to the utilization of research. This list indicates that the theory-practice gap operates in two directions: there is a lack of understanding amongst researchers of clinical issues and thus there may be a mismatch between the perceived value of research findings from the perspective of the researcher and their application in practice. Furthermore, there is a lack of understanding amongst practitioners of the mechanisms of research and the subsequent relevance of findings to their practice (Bryar, 1999).

We have already in our introduction alluded to the opportunity provided by PHC teams and their potential to develop into a research team. The NHS has increasingly recognized the relevance and importance of primary care research (Culyer, 1994) and as a result a more optimistic and positive climate for primary care multidisciplinary research has emerged. The opportunity for multidisciplinary teams to identify relevant, important and interesting research questions and pursue them in a supportive environment has never been better. With the regional research offices already thinking about transferring responsibilities of primary care research to local PCOs in the near future, local multidisciplinary research can put itself on the map by ensuring communication is established between themselves, research networks, other research bodies and the PCOs.

\section{Multidisciplinary research - practical issues}

Despite the potential value of a piece of successful multidisciplinary research, this can be very difficult to pursue and complete for all the reasons mentioned above. We would like to suggest several ideas, some conceptual and others more practical, which may help to create the infrastructure for multidisciplinary research teams in primary care.

To begin with, research may simply be seen as an extension of audit thereby making it less threatening for all members of the primary health care Press 
team. Ideas can be picked up and developed initially inhouse with support from local networks as happens in Hull where the Wolds Research Network (WoReN) offers practical advice on how research ideas may be progressed. Research practices might also have a supporting role in helping neighboring practices with their research ideas. Many research questions important in primary care do not require major financial or other resources, but where they do again local networks can provide support. We also need to integrate the development of research ideas into daily practice (Haynes and Haines, 1998).

\section{Moving forward}

If we truly believe that a research-conscious multidisciplinary workforce is important in primary care then it would be possible to support research development from general medical services funding through quality framework payments linked to clinical governance or even tiered payments such as those used historically to fund chronic disease management programs rewarding different levels of activity. The new contract for general practice could provide similar opportunities. Such activities have also been successfully implemented for performing minor surgery and incentivized prescribing. Indeed the results of local research might be used to inform standards and so influence payments and incentives.

Primary care organizations are well placed to support the development of multidisciplinary research working in primary care. As organizations, their structures reflect the various professionals involved in primary care and offer the opportunity for an inclusive approach to local research. They should be encouraged to develop their own research structures that promote multidisciplinarity.

\section{Collaboration in research}

There is a considerable body of research that has explored the difficulties that nurses have in being associated with research and research teams. The main mechanisms identified come under the headings of culture, interest and support (Closs and Cheater, 1996). Culture refers to societal and organizational support for research. Interest is concerned with stimulating the interest of practitioners in research through education about research, through researchers writing in ways that are interesting to practitioners and through making research findings accessible. Support is concerned with the mechanisms for helping the adoption of research into practice. The advent of research practices has given the opportunity for such practices to implement and practice the above mentioned concepts through collaboration (Pereira-Gray, 1995). Linking of research practices between each other clearly has the advantage of bringing together primary healthcare practitioners from both practices and providing the opportunity for training and learning between each other as well as in disseminating this knowledge to other practices and primary care professionals (Figure 4).

\section{Transforming multidisciplinary research questions into active research}

In order to illuminate what actually happens within a multidisciplinary research team, we provide two examples.

\section{Example one}

One research project in progress concerns the delivery of care to patients with serious enduring mental health problems such as schizophrenia. A paper (Burns and Kendrick, 1997) generated discussion within the practice that we then shared with our local community mental health team. A research question came out of our discussions and a group organized to develop a method to provide an answer. The team comprised practice nurses, community psychiatric nurses and a GP who consulted a number of resources including psychiatrists, a qualitative researcher from the research network and a statistician. With the exception of the GP researcher all the team members were new to research and the team used a problem-based approach developing the required research skills as the project went along. In this way the group explored literature searching, questionnaire design, interviews and their qualitative analysis, health status measurement tools, ethics and research project management amongst other research skills. Each discipline, aware of the clinical requirements of providing care to patients with chronic mental health problems, could ensure that their own professional skills and needs were taken 
- When and how far do we investigate unexpected findings of mildly abnormal liver function tests?

- What is the evidence that spirometry in primary care is superior to clinical standards, including peak flow monitoring for the diagnosis of airways disease?

- What may be the reasons for persistent failure of one practice to attain the higher target for preschool immunization despite all efforts?

- How do the differences in consultation style of general practitioners within a single practice influence the appointment system?

- Practice-based learning for all staff and nurses in basic aspects of researchhow achievable are they?

- Delivery of care to patients with schizophrenia in primary care

Figure 4 Examples of research questions following multidisciplinary collaboration between two research practices

into account in both planning and researching a new general practice-based service. The research question finally generated was 'Is the clinical care and outcome of patients with serious mental heath problems improved by joint community psychiatric nurse/practice nurse working in a general practice setting?' The project has now reached a stage where it is ready to be implemented (see Figure 5 for summary).

\section{Example two}

The difficulty of diagnosing airways disease in people over the age of 45 resulted in two GPs and two practice nurses from two different practices forming a research team. Both nurses had never been involved in research before but were actively interested in taking part as they perceived that the research process would directly benefit patients in the short and long term, as well as giving them

- Paper generated discussion between general practitioners and practice nurses

- Idea shared with practice nurses and community psychiatric nurses, consultant psychiatrist, a qualitative researcher and statistician

- A multidisciplinary team is formed

- Skills pooled together to develop proposal

- Shared ownership and direct impact on patient care

- Most professionals new to research but learn through a problem based approach and sharing of knowledge

- Project ready to be implemented with minimal or no funding

Figure 5 Schizophrenia research 
the opportunity to learn about research through a problem-based approach. Several research meetings took place and many were attended by the research facilitator from the local research network and a statistician. Expert advice was obtained from the local primary care academic department, as well as two chest physicians. The final research question was 'Does the addition of spirometry make any difference to the final diagnosis of airways obstruction'? The proposal took 18 months to complete and was agreed by all professionals involved. Funding from a non-NHS organization was quickly obtained and the project completed within 12 months. An abstract from this research has been presented at the British Thoracic Society Winter meeting (Raghunath et al., 2001) and the entire multidisciplinary team is writing up the full paper. The study process was also presented at a problem-based research workshop organized by the local research network (see Figure 6 for summary).

\section{Making collaboration work}

Our experience of multidisciplinary research working suggests certain ingredients facilitate effective collaborative approach. First amongst these is arriving at a shared perspective of what the problems are in clinical practice as experienced by individual team members. Valuing the contributions of everyone involved and creating a sense of shared ownership provides a rich environment to explore and develop research ideas. Providing protected time, training and support will allow the team to achieve its research goals. It is not sufficient however to simply set up a collaborative research team without investing in time and effort to manage and maintain that collaboration. This in itself requires time and our most recent development has therefore been the appointment of research facilitator to co-ordinate and support ongoing research between our two practices.

\section{Assessment of research in primary care and research governance}

If multidisciplinary research in primary care is to flourish then its external assessment will need to be carefully considered (Carter and Shaw, 1998). There appears to be a gap in the understanding and perception of 'success' in research terms between those conducting the research and fund providers. It takes time for multidisciplinary research work to develop and investment now is very much for future rewards. Equally however, there is a need to demonstrate accountability and value for money. Value for money is the most difficult area to assess and its assessment, like multidisciplinary research methodology itself, requires new thinking. What value for example might you attach to the development of a research protocol by a primary health

- Practical difficulty of diagnosis discussed between general practitioners/nurses

- Nurses learnt about research through problem-based and needs based approach as well as sharing of skills and knowledge

- Specific research needs identified and resources obtained through local research network, chest physicians and primary care academic department

- Research team developed proposal over a 18 month period

- Funding successfully obtained and project completed within the stipulated time

- Write up in progress, abstracts submitted and accepted by the BTS

- Patients enjoyed taking part and directly benefited

- Research findings implemented into practice and disseminated

- The research team felt motivated to undertake further multidisciplinary research 
care team that takes a year or more to produce but does not receive funding and is never carried out? Although the project ultimately fails much is gained in terms of research experience by the team itself. How do you value a small scale piece of research whose conclusions are important and useful to a particular primary health care team, but not generalizable to others? A recent evaluation of the primary care research practice initiative in the Northern and Yorkshire region (Gray, 2000) felt strongly that the scheme had been 'good value for money' but struggled to describe this in objective terms. It is surely the case that the assessment of research in primary care as it becomes more mainstream needs to take account of research activity and processes as much as output.

Just as clinical governance seeks to ensure quality in clinical practice, research governance has been recently introduced to ensure quality of research. Multidisciplinary research teams are in a unique position to ensure self-assessment and monitoring of their research activities through mutual information sharing and application of the governance process at every stage of research. Knowledge gained from multidisciplinary research teams should help health professionals to inform and apply research governance in their own working organization.

\section{Multidisciplinary research education and training}

If the culture of research is to really become part of the daily life of primary care professionals then research must become an integral part of training. There is already a move towards this in vocational training and community nursing degree courses now include an introduction to research methods. These initial steps need to be developed with continuing encouragement to continue research interests as practitioners make the transition from trainee to independent professionals. Being a part of a multidisciplinary team is as mentioned earlier, itself an educational and training process. Often such problem-based learning is all that may be required for most health professionals trying to balance service commitments and research inter- ests. Multidisciplinary research teams as we have demonstrated in our examples can ensure that research, educational and training needs of different health professionals are appropriately recognized and met. Thus enthusiasm can be maintained in individuals without risk of 'burn-out' who can then pass on their learnt skills to newcomers to research teams thus creating a spiraling increase in research capacity. Some research practices will develop into self-sufficient research organizations and will be in a unique position to promote such training and education both within and outside the organization. We believe that they could act as 'mini-research networks' that are inherently in tune with workings of health professionals and their research training needs within a multidisciplinary set-up.

\section{Conclusions}

Primary care research is developing rapidly with much progress made in supporting development of research outside academic units. The future of service-based primary care research with its rewards of a research-conscious workforce able to undertake and apply research in their daily practice lies with PCOs and these new organizations offer the opportunity for research to be targeted at locally important questions in a way not previously realized. Primary care group boards were dominated by a multidisciplinary professional team inherently in tune with their practicing colleagues. As primary care trusts (PCTs) have come on stream with a more management-centered board structure we need to make sure that they remain aware both of need to keep research and development at the forefront of their planning and also to support a multidisciplinary framework for it to flourish.

In summary, we believe that the development of multidisciplinary research depends on research becoming an integral part of multidisciplinary primary care working funded perhaps through general medical services budgets and supported by PCOs that are capable of accepting their responsibilities for primary care research and development (Charles-Jones, 2000, Thomas et al., 2000). (Figure 7) 
- A broad base of primary care professionals and practices need to be identified.

- Multidisciplinary research teams need support and facilitation.

- Research needs to be envisaged as a part of day-to-day work of primary care.

- Context based research questions may be more important and acceptable.

- New approaches to funding practices including general medical services linked funding needs to be considered.

- Combining pragmatism with quality is important.

- Training needs to be tailored to individual needs.

- PCOs must ensure that multidisciplinary research has a high priority

Figure 7 Requirements for multidisciplinary research in primary care to succeed

\section{Acknowledgements}

The authors are research leads in NHSE sponsored research practices, which collaborate closely in their research activity.

\section{References}

Beasley, J. 1993: The structure and activity of primary care research networks. Family Practice Research Journal 13, 395-403.

Bryar, R. 1999: The transition of practitioner to practitioner researcher in primary health care. $\mathrm{PhD}$ thesis. Cardiff: University of Wales College of Medicine.

Burns, T. and Kendrick, T. 1997: The primary care of patients with schizophrenia: a search for good practice. British Journal of General Practice 47, 515-19.

Carter, Y. and Shaw, S. 1998: Accrediting research in general practice. British Journal of General Practice 48, 171-74.

Carter, Y. and Thomas, C., editors, 1997: Research methods in primary care. Oxford: Radcliffe Medical Press.

Charles-Jones, H. 2000: Primary care groups and research. British Journal of General Practice 50, 505.

Closs, S. and Cheater, F. 1996: Audit or research: what is the difference? Journal of Clinical Nursing 5, 249-56.

Coulter, A. 1999: Paternalism or partnership. British Medical Journal 319, 719-20.

Culyer, A. 1994: Supporting research and development in the NHS: a report to the minister for health by a research and development task force chaired by Professor Anthony Culyer. London: HMSO.

English, I. 1994: Nursing as a research based profession: 22 years after Briggs. British Journal of Nursing 3, 402-06.
Goodare, H. and Lockwood, S. 1999: Involving patients in clinical research. British Medical Journal 319, 724-25.

Gray, S. 2000: Evaluation of the research practice scheme. Durham: Northern and Yorkshire NHS Executive.

Griffiths, F. and Byrne, D. 1998: General practice and the new science emerging from the theories of chaos and complexity. British Journal of General Practice 48, 1697-99.

Haynes, B. and Haines, A. 1998: Getting research findings into practice: barriers and bridges to evidence based clinical practice. British Medical Journal 317, 273-76.

Hicks, C. 1995: The shortfall in published research: a study of nurses' research and publication activities. Journal of Advanced Nursing 21, 594-604.

Hippisley-Cox, J. and Pringle, M. 1997: Helicobacter pylori: a pilot study of a randomized control trial of pragmatic eradication. British Journal of General Practice 47, 375-77.

Hobbs, R. 1997: Why do research in primary care? In Thomas, C., editors, Research methods in primary care. Oxford: Radcliffe Medical Press, 1-7.

Kernick, D., Stead, J. and Dixon, M. 1999: Moving the research agenda to where it matters. British Medical Journal 319, 206-07.

Mant, D. 1997: National working group on R\&D in primary care: final report. London: NHS Executive.

McKenzie, A. 1991: Nurses in research in primary health care. Nursing RSA 6, 26-27.

Mooney, M. 1996: Primary health care research: what it is and what it is not. The Nurse Practitioner 21, 8-15.

Pereira-Gray, D. 1995: Research general practices. British Journal of General Practice 5, 516-17.

Radda, G. 1998: Primary care research: the MRC's proposals. British Journal of General Practice 48, 872.

Raghunath, A., Innes, A., Norfolk, L., Hannant, M., Greene, T., Greenstone, M. and Morice, A. 2001: A comparison of 
primary care vs. specialist diagnosis of airways disease. Thorax 56, 87-88.

Shaw, S. and Carter, Y. 2000: RCGP is encouraging improvements in primary care research. British Medical Journal 320, 314.

Thomas, P., Kai, J., Griffiths, F. and O'Dwyer, A. 2000: Primary care groups and research networks: opportunities for R\&D in context. British Journal of General Practice 50, 91-92.

Thomas, P. and While, A. 2001: Increasing research capacity and changing the culture of primary care towards reflective practice: the experience of the West London Research Network (WeLReN). Journal of Interprofessional Care 15, 133-39. van der Windt, D., Koes, B., van Aarst, M, Heemskerk, M. and Bouter, L. 2000: Practical aspects of conducting a pragmatic randomized trial in primary care: patient recruitment and outcome assessment. British Journal of General Practice 50, 371-74.

Whitford, L., Jelly, D., Gandy, S., Souther, A. and Van Zwaneberg, T. 2000: Making research relevant to the primary health care team. British Journal of General Practice 50, 573-76.

WHO. 1994: Nursing beyond the year 2000. A report of a WHO study group. Geneva: WHO.

Williamson, C. 1999: The challenge of lay partnerships. British Medical Journal 319, 721-22. 